\title{
NONUNIFORM DECIMATION AND RECONSTRUCTION OF GENERALIZED-BANDLIMITED MD SIGNALS
}

\author{
Tsuhan Chen and P. P. Vaidyanathan \\ Department of Electrical Engineering, 116-81 \\ California Institute of Technology \\ Pasadena, California 91125, USA
}

\begin{abstract}
It is well known that the data rate of bandlimited signals can be reduced without loss of information. One scheme which achieves this goal is the so-called nonuniform decimation. Recent results show that a bandlimited signal can be reconstructed from a nonuniformly decimated version. Theoretical results and efficient reconstruction methods have been addressed for one-dimensional signals. For the multidimensional case, some partial results are known. In this paper, we will discuss in detail the theory and implementation of the reconstruction of generalizedbandlimited multidimensional signals from their nonuniformly decimated versions.
\end{abstract}

\section{INTRODUCTION}

For discrete-time bandlimited signals, the data rate can be reduced without losing any information. The reason of this can be traced back to the fundamental sampling theorem of Shannon and its various extensions [1]. One of the schemes that achieve such reduction is the uniform rational decimation [2], [3]. Suppose $x(n)$ is bandlimited to the region $-L \pi / M \leq \omega<L \pi / M$. Rational decimation can reduce the data rate by $M / L$ without losing information. Rational decimation has also been extended to the multidimensional (MD) case in [4], [5].

Nonuniform decimation is another method of compressing such bandlimited signals [6]. Fig. 1 shows an MD nonuniform decimation and reconstruction system. With $D$ denoting the number of dimensions, the decimation factor $\mathbf{M}$ in this system is a nonsingular $D \times D$ integer matrix. The terms $e^{-j \omega^{T} \mathbf{n}_{\ell}}$ are MD shift operators and $F_{i}(\omega)$ are MD filters. Only $L$ out of $M$ polyphase components of $x(\mathbf{n})$ are retained and transmitted to the synthesis end, where $M=|\operatorname{det} \mathbf{M}|$. We want to find synthesis filters $F_{k}(\omega)$ such that $x(\mathbf{n})$ is

\footnotetext{
Work supported in parts by the NSF grant MIP 8919196 , and by matching funds from Tektronix, Inc., and Rockwell International.
}

perfectly reconstructed, i.e., $\widehat{x}(\mathbf{n})=x(\mathbf{n})$. One main advantage of this data compression scheme is that the compression part is very simple and all the arithmetic operations are only in the reconstruction part. For the one-dimensional (1D) case, is has been shown that perfect reconstruction is possible if $x(n)$ is bandlimited [6]. The synthesis filters $F_{k}(z)$ that achieve perfect reconstruction have piecewise-constant frequency responses, and are called multilevel filters. An efficient implementation of such filters has also been derived in [6]. When $\mathbf{M}$ is diagonal, some results for the two-dimensional (2D) have been derived in [7]. For the general 2D case, some partial results are also known [8].

To extend $1 \mathrm{D}$ existing results to the general MD case, one has to generalize the idea of 'bandlimitedness' first. In this paper, we will discuss this in detail. We will first present an example which shows the feasibility of MD nonuniform decimation and reconstruction. Then, we discuss the design of synthesis filters for the general case. We will also address the conditions that determine the feasibility of perfect reconstruction and the efficient implementation of synthesis filters.

Notations and Preliminaries: In this paper, we use the same notations as in [9], [10]. Refer to [9], [10] for basic concepts of MD multirate systems. In particular, if an MD signal $x(\mathbf{n})$ is decimated by $\mathbf{M}$ and then expanded by $\mathbf{M}$ to produce the signal $y(\mathbf{n})$, the corresponding frequency domain relation is

$$
Y(\omega)=\frac{1}{M} \sum_{i=0}^{M-1} X\left(\omega-\omega_{i}\right)
$$

where $\omega_{i}=2 \pi \mathbf{M}^{-T} \mathbf{k}_{i}$, and $\mathbf{k}_{i} \in \mathcal{N}\left(\mathbf{M}^{T}\right)$, which is the set of all integer vectors of the form $\mathbf{M}^{T} \mathbf{x}, \mathbf{x} \in[0,1)^{D}$. The vectors $\omega_{i}$ will be called the aliasing offsets. The 0 -th offset $\omega_{0}$ is chosen as the zero vector 0 . 


\section{AN EXAMPLE}

We shall start with the $2 \mathrm{D}$ example where

$$
\mathbf{M}=\left[\begin{array}{cc}
1 & 1 \\
2 & -2
\end{array}\right]
$$

which defines the so-called hexagonal decimation. Corresponding to this $\mathbf{M}$, the alias offsets are

$\omega_{0}=\left[\begin{array}{l}0 \\ 0\end{array}\right], \omega_{1}=\left[\begin{array}{c}\pi \\ \pi / 2\end{array}\right], \omega_{2}=\left[\begin{array}{l}0 \\ \pi\end{array}\right], \omega_{3}=\left[\begin{array}{c}\pi \\ 3 \pi / 2\end{array}\right]$.

With respect to this $\mathbf{M}$, the frequency domain can be partitioned into four regions $\mathcal{R}_{i}$, with each $\mathcal{R}_{i}$ being a hexagon centered at one alias frequency $\omega_{i}$, as in Fig. 2. Note that the Fourier transform of an MD signal is periodic in each dimension with period $2 \pi$. Suppose that the spectrum $X(\omega)$ of an MD signal is bandlimited in regions $\mathcal{R}_{0}$ and $\mathcal{R}_{2}$. We now show that by keeping only 2 out of every 4 samples of $x(n)$, we can recover the whole signal $x(\mathbf{n})$.

Let us keep only two coset members $x(\mathbf{M n})$ and $x\left(\mathbf{M n}-\left[\begin{array}{ll}1 & 1\end{array}\right]^{T}\right)$, as shown in Fig. 3. In other words, two out of four polyphase components [9], [10] of $x(\mathbf{n})$ are kept. This can be realized as in Fig. 1 with $\mathbf{n}_{0}=\left[\begin{array}{ll}0 & 0\end{array}\right]^{T}$ and $\mathrm{n}_{1}=\left[\begin{array}{ll}1 & 1\end{array}\right]^{T}$. Now, we want to design the synthesis filters $F_{0}(\omega)$ and $F_{1}(\omega)$ to reconstruct the original signal, i.e., to make $\widehat{x}(\mathbf{n})=x(\mathbf{n})$.

Using (1), we can obtain the Fourier transform of $v_{0}(\mathbf{n})$ in terms of $X(\boldsymbol{\omega})$

$V_{0}(\omega)=\frac{1}{4}\left[X(\omega)+X\left(\omega-\omega_{1}\right)+X\left(\omega-\omega_{2}\right)+X\left(\omega-\omega_{3}\right)\right]$.

Similarly, we get

$$
\begin{aligned}
V_{1}(\omega) & =\frac{1}{4}\left[e^{-j \omega^{T} \mathbf{n}_{1}} X(\omega)\right. \\
& +e^{-j\left(\omega^{T} \mathbf{n}_{1}+\omega_{1}^{T} \mathbf{n}_{1}\right)} X\left(\omega-\omega_{1}\right) \\
& +e^{-j\left(\omega^{T} \mathbf{n}_{1}+\omega_{2}^{T} \mathbf{n}_{1}\right)} X\left(\omega-\omega_{2}\right) \\
& \left.+e^{-j\left(\omega^{T} \mathbf{n}_{1}+\omega_{3}^{T} \mathbf{n}_{1}\right)} X\left(\omega-\omega_{3}\right)\right]
\end{aligned}
$$

Because $X(\omega)$ is bandlimited in the regions $\mathcal{R}_{0}$ and $\mathcal{R}_{2}$, it is reasonable to let $F_{0}(\omega)$ and $F_{1}(\omega)$ be zero for $\omega \in \mathcal{R}_{1} \cup \mathcal{R}_{3}$. Also owing to the fact that $X(\omega)$ is bandlimited, when $\omega \in \mathcal{R}_{0}$, we get

$$
\begin{aligned}
V_{0}(\omega) & =\frac{1}{4}\left[X(\omega)+X\left(\omega-\omega_{2}\right)\right], \\
V_{1}(\omega) & =\frac{1}{4} e^{-j \omega^{T} \mathbf{n}_{1}}\left[X(\omega)+e^{-j \omega_{2}^{T} \mathbf{n}_{1}} X\left(\omega-\omega_{2}\right)\right],
\end{aligned}
$$

When $\omega \in \mathcal{R}_{2}$, we get

$$
V_{0}(\omega)=\frac{1}{4}\left[X(\omega)+X\left(\omega-\omega_{2}\right)\right],
$$

$$
V_{1}(\omega)=\frac{1}{4} e^{-j \omega^{T} \mathbf{n}_{1}}\left[X(\omega)+e^{-j \omega_{2}^{T} \mathbf{n}_{1}} X\left(\omega-\omega_{2}\right)\right] .
$$

It is interesting to note that we get the same expressions for both $\mathcal{R}_{0}$ and $\mathcal{R}_{2}$. Noticing that $e^{-j \omega_{2}^{T} \mathbf{n}_{1}}=$ -1 , we obtain

$\widehat{X}(\omega)=\frac{1}{4}\left[X(\omega) X\left(\omega-\omega_{2}\right)\right]\left[\begin{array}{cc}1 & 1 \\ 1 & -1\end{array}\right]\left[\begin{array}{c}F_{0}(\omega) \\ e^{-j \omega^{T} \mathbf{n}_{1}} F_{1}(\omega)\end{array}\right]$

for $\omega \in \mathcal{R}_{0} \cup \mathcal{R}_{2}$. Therefore, for $\widehat{X}(\omega)=X(\omega)$, we need to have

$$
\frac{1}{4}\left[\begin{array}{cc}
1 & 1 \\
1 & -1
\end{array}\right]\left[\begin{array}{c}
F_{0}(\omega) \\
e^{-j \omega^{T} \mathbf{n}_{1}} F_{1}(\omega)
\end{array}\right]=\left[\begin{array}{l}
1 \\
0
\end{array}\right],
$$

which implies

$$
F_{0}(\omega)=2 \quad \text { and } \quad F_{1}(\omega)=2 e^{j \omega^{T} \mathbf{n}_{1}} .
$$

Since $F_{0}(\omega)$ and $F_{1}(\omega)$ are zero for $\omega \in \mathcal{R}_{1} \cup \mathcal{R}_{3}$, the resulting nonuniform decimation and reconstruction system can be implemented as in Fig. 4, where $H_{L}(\omega)$ is a lowpass filter having passband support exactly the same as the support of $X(\omega)$. In other words, to reconstruct $x(\mathbf{n})$, we only have to pass the nonuniform decimated version of $x(\mathbf{n})$ through the lowpass filter $H_{L}(\omega)$ !

We use the 'Lenna' image to test this system. We prefilter the image such that it is bandlimited in $\mathcal{R}_{0} \cup$ $\mathcal{R}_{2}$. The 'bandlimited Lenna' is shown in Fig. 5(a). Using the system in Fig. 4, we obtain the 'reconstructed Lenna' in Fig. 5(b). The resulting signal to noise ratio is $43.8 \mathrm{~dB}$. Note that the reconstruction error is due to the fact that $H_{L}(\omega)$ is not an ideal filter. Theoretically speaking, the original bandlimited would be perfectly reconstructed if $H_{L}(\omega)$ were ideal.

\section{MORE GENERAL RESULTS}

Consider the general MD nonuniform decimation and reconstruction system shown in Fig. 1. Without loss of generality, one can let $\mathbf{n}_{i} \in \mathcal{N}(\mathbf{M})$. In the $\ell$-th channel, those $x(\mathbf{n})$ where $\mathbf{n}+\mathbf{n}_{\ell} \in L A T(\mathbf{M})$ are retained. Hence, $L$ out of $M$ polyphase components of $x(\mathbf{n})$ are kept. Using (1), one can verify that

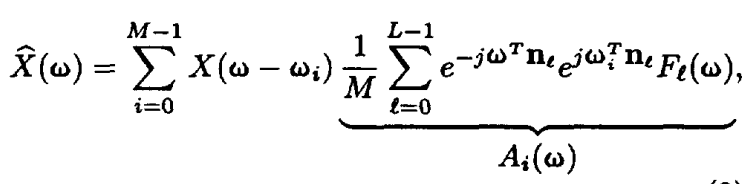

where $\omega_{i}$ are the aliasing offsets with respect to the decimation matrix M. From this, we see that we need 
$A_{0}(\omega)=1$ and $A_{i}(\omega)=0$ for $i \neq 0$ to achieve perfect reconstruction.

Suppose the MD frequency domain can be partitioned into $M$ regions $\mathcal{R}_{0}, \mathcal{R}_{1}, \ldots, \mathcal{R}_{M-1}$. All these regions have the same shape and each of them is associated with one aliasing offset. More specially, these regions can be expressed as $\mathcal{R}_{0}+\omega_{i}, 0 \leq i \leq M-1$. Suppose the input spectrum $X(\omega)$ is nonzero only in $L$ out of these $M$ regions, say $\mathcal{R}_{0}, \mathcal{R}_{1}, \ldots, \mathcal{R}_{L-1}$. We say that $X(\omega)$ is generalized-bandlimited.

Since $X(\omega)$ is bandlimited in $\mathcal{R}_{0}, \mathcal{R}_{1}, \ldots, \mathcal{R}_{L-1}$, we can let all $F_{\ell}(\omega)$ be zero for $\omega \in \mathcal{R}_{L} \cup \cdots \cup \mathcal{R}_{M-1}$. Moreover, because $X(\omega)$ is bandlimited, it can be verified that at most $L$ terms of $X\left(\omega-\omega_{i}\right)$ in (9) can be nonzero at a certain $\omega$. For example, when $\omega \in \mathcal{R}_{0}$, only $X\left(\omega+\omega_{i}\right)$ for $0 \leq i \leq L-1$ are nonzero. Therefore, from (9), we get

$$
\begin{aligned}
& \underbrace{\left[\begin{array}{ccc}
e^{\omega_{0}^{T} \mathbf{n}_{0}} & \cdots & e^{\omega_{0}^{T} \mathbf{n}_{L-1}} \\
\vdots & \ddots & \vdots \\
e^{\omega_{L-1}^{T} \mathbf{n}_{0}} & \cdots & e^{\omega_{L-1}^{T} \mathbf{n}_{L-1}}
\end{array}\right]}_{\mathrm{V}}\left[\begin{array}{c}
F_{0}(\omega) \\
\vdots \\
e^{-j \omega^{T} \mathbf{n}_{L-1}} F_{L-1}(\omega)
\end{array}\right] \\
& =M\left[\begin{array}{lll}
A_{0}(\omega) & \cdots & A_{L-1}(\omega)
\end{array}\right]^{T} .
\end{aligned}
$$

We want to choose the filters $F_{\ell}(\omega)$ such that $A_{0}(\omega)=$ 1 and $A_{i}(\omega)=0$ for $i \neq 0$. If the $L \times L$ matrix in (10) is nonsingular, we can solve for $F_{\ell}(\omega)$ in the region $\mathcal{R}_{0}$. Clearly, each $F_{\ell}(\omega)$ will have a constant value in $\mathcal{R}_{0}$. Repeatedly doing this for other regions $\mathcal{R}_{1}, \ldots, \mathcal{R}_{L-1}$, we can obtain the required filter responses for all $\omega$. It can be verified that all $e^{-j \omega^{T} n_{\ell}} F_{\ell}(\omega)$ have piecewiseconstant frequency responses. These are called multilevel filters.

Singularity of $\mathbf{V}$ : The matrix $\mathrm{V}$ is a submatrix of the generalized discrete Fourier transform (DFT) matrix with respect to $\mathbf{M}$ [10]. For the special case that the vectors $\mathbf{n}_{\ell}$ can be expressed as $k \mathbf{m}$ (modulo $\mathbf{M}$ ) for $0 \leq k \leq L-1, \mathrm{~V}$ becomes a Vandermonde matrix. In this case, $\mathrm{V}$ is nonsingular if and only if all $\omega_{i}^{T} \mathbf{m}$ are distinct.

\section{EFFICIENT RECONSTRUCTION}

For the $1 \mathrm{D}$ case, it has been shown the reconstruction filters can be obtained at the cost of one $M$-th band lowpass filter and a constant matrix multiplier [6]. We extended this to the MD case.

Assume that the MD frequency domain is partitioned as described in the previous section. Consider a multilevel MD filter $F(\omega)$ which has complex value $\beta_{i}$ in the region $\mathcal{R}_{i}, 0 \leq i \leq L-1$, and zero otherwise.
So,

$$
F(\omega)=\sum_{\ell=0}^{L-1} \beta_{\ell} B\left(\omega-\omega_{\ell}\right),
$$

where $B(\omega)$ is unity in $\mathcal{R}_{0}$ and zero otherwise. Now, consider the filter $H(\omega)$ which is unity in $\omega \in \mathcal{R}_{0} \cup$ $\ldots \cup \mathcal{R}_{L-1}$ and zero otherwise, so that

$$
H(\omega)=\sum_{\ell=0}^{L-1} B\left(\omega-\omega_{\ell}\right) .
$$

Compute the polyphase decomposition [9], [10] of $B(\omega)$ with respect to $\mathbf{M}$

$$
B(\boldsymbol{\omega})=\sum_{i=0}^{M-1} e^{-j \omega^{T} \mathbf{n}_{i}} B_{i}\left(\mathbf{M}^{T} \omega\right),
$$

where $\mathbf{n}_{i} \in \mathcal{N}(\mathbf{M})$. Substituting (13) into (12), we get

$$
\begin{aligned}
H(\omega) & =\sum_{\ell=0}^{L-1} \sum_{i=0}^{M-1} e^{-j \omega^{T} \mathbf{n}_{i}} B_{i}\left(\mathbf{M}^{T}\left(\omega-\omega_{\ell}\right)\right) e^{-j \omega_{\ell}^{T} \mathbf{n}_{i}} \\
& =\sum_{i=0}^{M-1} e^{-j \omega^{T} \mathbf{n}_{i}} B_{i}\left(\mathbf{M}^{T} \omega\right) \underbrace{\sum_{\ell=0}^{L-1} e^{-j \omega_{\ell}^{T} \mathbf{n}_{i}}}_{q_{i}} \cdot(14)
\end{aligned}
$$

So, we see that $H_{i}(\omega)=B_{i}(\omega) q_{i}$ are the polyphase components of $H(\omega)$. Similarly, we can obtain

$$
F(\omega)=\sum_{i=0}^{M-1} e^{-j \omega^{T} \mathbf{n}_{i}} H_{i}\left(\mathbf{M}^{T} \omega\right) \frac{\alpha_{i}}{q_{i}}
$$

where $\alpha_{i}=\sum_{\ell=0}^{L-1} \beta_{\ell} e^{-j \omega_{\ell}^{T} \mathbf{n}_{i}}$, assuming that all $q_{i}$ are nonzero. So, we conclude that a multilevel filter can be obtained from the polyphase components of the filter $H(\omega)$.

Hence, a bank of $L$ multilevel filters can be implemented at the cost of one filter $H(\omega)$ and several constant multipliers. This results in the complete synthesis bank shown in Fig. 6, where $\mathbf{C}$ is an $M \times L$ constant matrix multiplier.

\section{CONCLUSIONS}

In this paper, we have discussed the nonuniform decimation and reconstruction of generalized-bandlimited MD signals. We presented an example which shows the feasibility of reconstructing an MD signal from its nonuniformly decimated version. Then, we extended this idea to the general case and discussed the theory and design of synthesis filters for reconstruction. We have also addressed the conditions for the feasibility of perfect reconstruction and the efficient polyphase implementation of synthesis filters. 


\section{REFERENCES}

[1] A. J. Jerri, "The Shannon sampling theorem-its various extensions and applications: A tutorial review," Proc. IEEE, pp. 1565-1596, November 1977.

[2] R. E. Crochiere and L. R. Rabiner, Multirate Digital Signal Processing, Prentice-Hall, 1983.

[3] P. P. Vaidyanathan, "Multirate digital filters, filter banks, polyphase networks, and applications: A tutorial," Proc. IEEE, pp. 56-93, January 1990.

[4] E. Dubois, "The sampling and reconstruction of time-varying imagery with application in video systems," Proc. IEEE, vol. 73, pp. 502-522, April 1985.

[5] T. Chen and P. P. Vaidyanathan, "On the choice of rational decimation systems for multidimensional signals," IEEE ICASSP, Minneapolis, April 1993.

[6] P. P. Vaidyanathan and V. C. Liu, "Efficient reconstruction of band-limited sequences from nonuniformly decimated versions by use of polyphase filter banks," IEEE Trans. on Acoustics, Speech, and Signal Processing, vol. 38, no. 11, pp. 1927-1936, November 1990.

[7] V. C. Liu and P. P. Vaidyanathan, "Compression of two-dimensional band-limited signals using sub-sampling theorems," Journal of IETE, vol. 34, no. 5 , pp. $416-422,1988$.

[8] V. C. Liu, "One- and two-dimensional digital multirate systems with applications in subsampling and band-limited signal reconstruction," Ph.D. thesis, Caltech, 1989.

[9] E. Viscito, and J. P. Allebach, "The analysis and design of multidimensional FIR perfect reconstruction filter banks for arbitrary sampling lattices," IEEE Trans. on CAS, pp. 29-41, January 1991.

[10] P. P. Vaidyanathan, Multirate Systems and Filter Banks. Englewood Cliffs, NJ: Prentice-Hall, 1993.

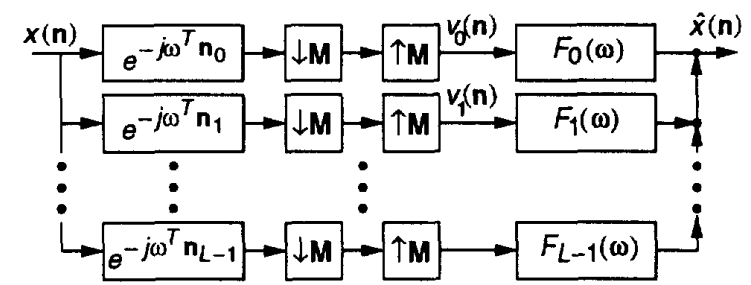

Fig. 1. Nonuniform decimation and reconstruction.

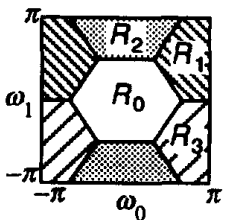

Fig. 2. Partition of the frequency domain.

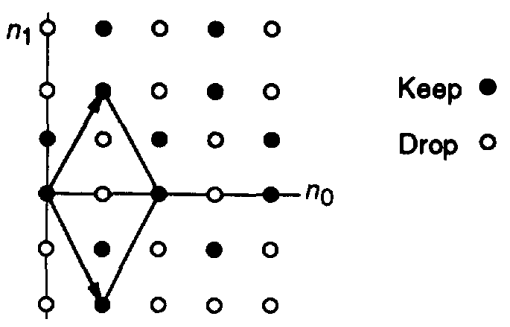

Fig. 3. Nonuniform decimation.

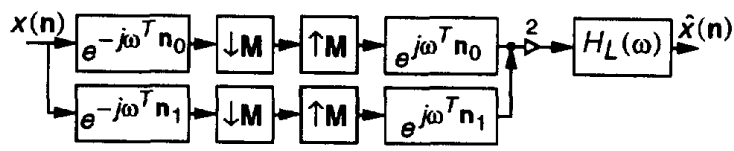

Fig. 4. Example in Section 2.

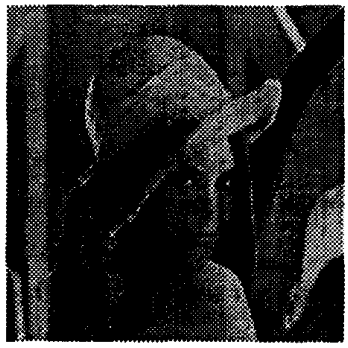

(a)

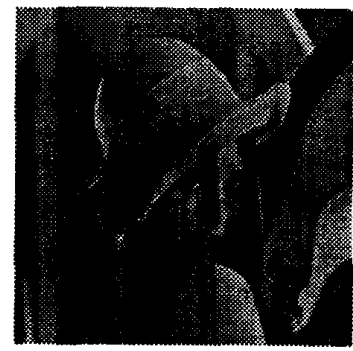

(b)
Fig. 5. (a) original bandlimited image, (b) reconstructed image.

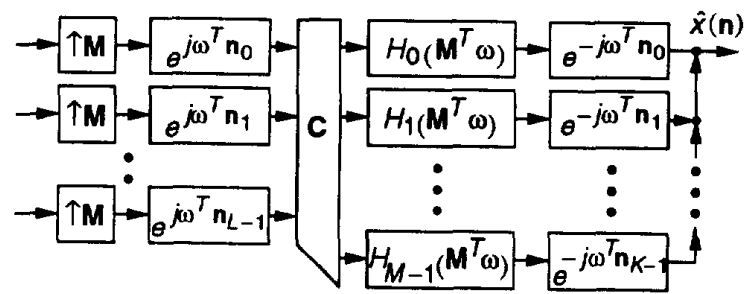

Fig. 6. Efficient reconstruction. 